\title{
Erratum: Lineage-specific chromatin signatures reveal a regulator of lipid metabolism in microalgae
}

Chew Yee Ngan, Chee-Hong Wong, Cindy Choi, Yuko Yoshinaga, Katherine Louie, Jing Jia, Cindy Chen, Benjamin Bowen, Haoyu Cheng, Lauriebeth Leonelli, Rita Kuo, Richard Baran, José G. García-Cerdán, Abhishek Pratap, Mei Wang, Joanne Lim, Hope Tice, Chris Daum, Jian Xu, Trent Northen, Axel Visel, James Bristow, Krishna K. Niyogi and Chia-Lin Wei Nature Plants 1, 15107 (2015); published online 27 July 2015; corrected 29 September 2015

In the version of this Article originally published, the author affiliations were incorrect. These have been corrected. 